\title{
UN TEMA DE TORRES NAHARRO Y DE GIL VICENTE
}

Ya se ha observado la semejanza que existe entre el tratamiento del tema de la Anunciación en el Diálogo del Nascimiento de Torres Naharro y en el Auto de Gil Vicente llamado da Mofina Mendes ${ }^{1}$. Antes de estudiar la cuestión, conviene poner a la vista del lector los pasajes esenciales de los dos textos. En primer lugar, he aquí el dẹ Torres Naharro:

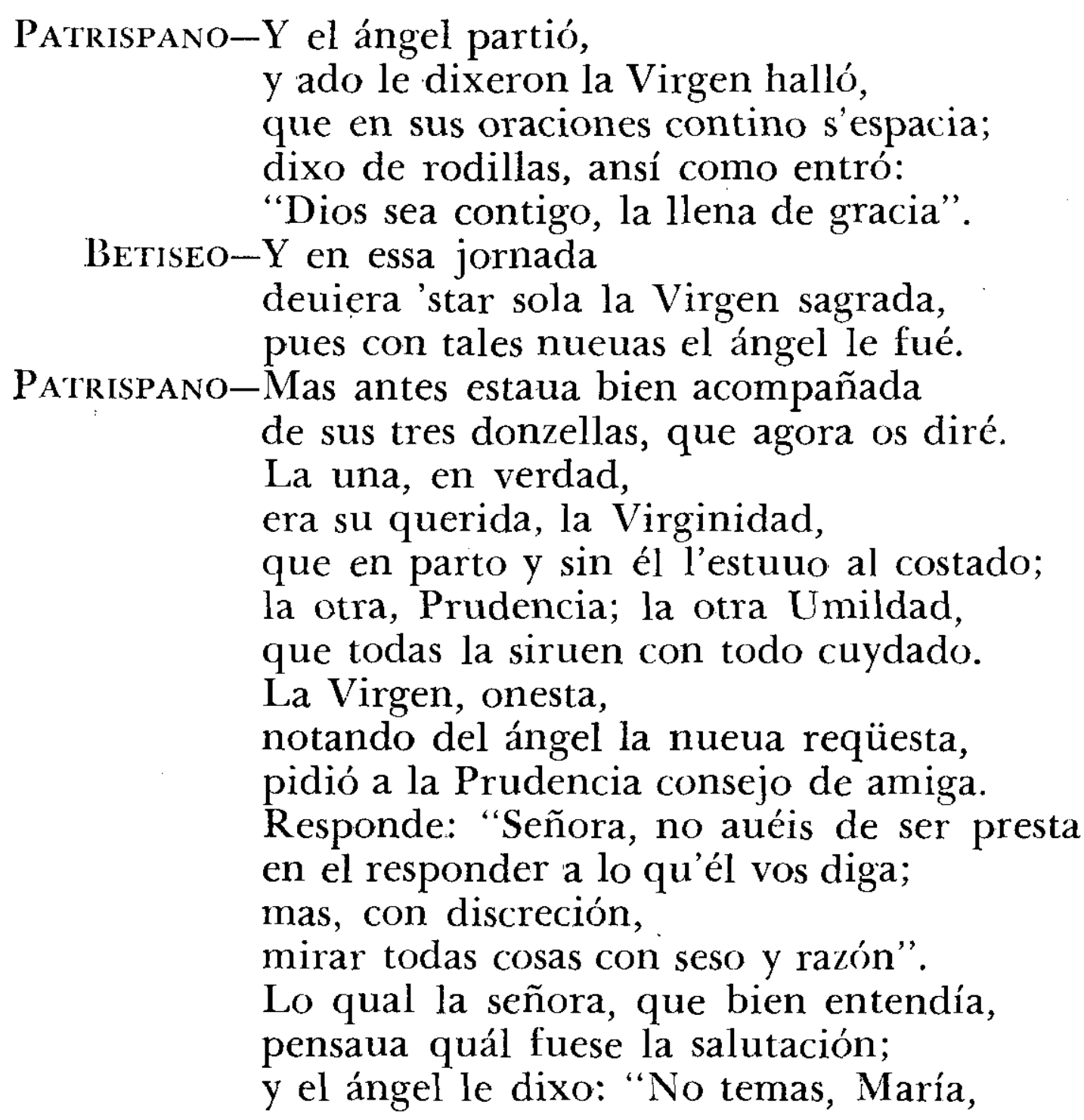

1 J. P. Wickersham Crawford, Spanish drama before Lope de Vega. A revised edition. Philadelphia, 1937, pág. 38: "The prophecies are related in some detail [en el Diálogo del Nascimiento], and also the Annunciation, which bears a strong resemblance to Gil Vicente's version of the same scene in the Auto da Mofina Mendes (1534)". 
que por tu valor

hallaste la gracia, vezina al Señor, y d'él un infante tú concibirás".

La Virgen, armada de sancto pudor, oyendo lo tal dudó mucho más;

y entonces, al ora,

la Virginidad le dixo: "Señora,

dí si por varón, o de qué manera.

Si dize que sí, tomemos agorà

y a palos le echemos por la puerta fuera".

Tornó con saber

la Virgen al ángel: “¿Cómo puede ser?, que nunca varón jamás conoscí".

Y el ángel le dixo: "Dios quiere [hazer]

qu'Espíritu Sancto venga sobre ti".

Y entonces María

dixo a la Humildad qué le parescía:

y aquélla responde, sin más altercar,

que aquello qu'el ángel ta[n] bien le dezía

con ojos humildes deuría aceptar.

La qual, con derecho,

dixo, las manos en cruz sobr'el pecho:

"Cata la sierua del alto Señor, yo soy muy contenta, y en mí sea hecho

según tu palabra, ta[n] bien y mejor" ".

En el auto de Gil Vicente, desde el Prólogo, el fraile predicador anuncia los personajes de la primera parte de la representación:

A qual obra he chamada os mysterios da Virgem, que entraraa acompanhada de quatro damas com quem de menina foy criada;

a hũa chamão Pobreza, outra chamão Humildade, damas de tanta nobreza que todalma que as preza he morada da Trindade.

A outra terceyra dellas chamão Fee per excelencia, aa outra chamam Prudencia, e viraa a Virgem com ellas

2 "Propalladia" and other works of Bartolomé de Torres Naharro, edited by Joseph E. Gillet, vol. I, Bryn Mawr, 1943, págs. 278-279. 
com muy fermosa aparencia:

sera logo o fundamento

tratar da saudação ...3

Cuando empieza la representación, la Virgen, rodeada de las cuatro Virtudes, estudia las profecías que sobre la Madre del Redentor formularon, según las creencias medievales, los Profetas de Israel y las antiguas Sibilas. En ese momento entra en escena el ángel $\mathrm{Ga}$ briel:

AnJo-Oo Deos te salue, Maria, chea de graça graciosa, dos peccadores abrigo: gozate com alegria, humana e diuina rosa, porque o Senhor he contigo.

VIrgem-Prudencia, que dizeis vos? que eu muyto turbada sam porque tal saudação nam se costuma antre nos.

Prudencia-Pois que he auco [sic] do Senhor, Senhora, nam esteis turbada, tornay em vossa color, que, segundo o embayxador, tal s'espera a embayxada.

Gabriel-Oo virgem, se ouuir me queres, mais te quero inda dizer: benta es tu em mereceres mais que todas as molheres nacidas e por nacer.

Virgem-Que dizeis vos, Humildade, qu'este verso vay muy fundo, porque eu tenho por verdade ser em minha calidade a menos cousa do mundo.

Humildade-O anjo que daa o recado sabe bem disso a certeza; diz Dauid, no seu tractado, que esse spirito assi humilhado he cousa que Deos mais preza.

Gabriel-Alta Senhora, saberaas que tua sancta humildade te deu tanta dignidade que hum filho conceberaas

"Copilagam de todalas obras de Gil Vicente, Lisboa, 1562 , fol. XXI. He'mos modificado la puntuación. 
da diuina eternidade.

Seu nome sera chamado Jesu e Filho de Deos e o teu ventre sagrado ficaraa orto çarrado e tu Princesa dos Ceos.

Virgem-Que direy, Prudencia minha? A vos quero por espelho. Prudencia-Segundo o caso caminha, deueis, Senhora Raynha, tomar com o anjo conselho.

VIRGEM-Quomodo fiet istud, quoniam virum non cognosco? Porque eu dei minha pureza ao Senhor e meu poder com toda minha firmeza.

GABRIEL-Spiritus sanctus superueniet in te. E a virtude do Altissimo, Senhora, te cubriraa, porque Seu Filho sera e teu ventre sacratissimo per graça conceberaa.

VIRGEM-Fee, dizey-me vosso intento, que este passo a vos conuem; cuydemos nisto muy bem, porque a meu consentimento grandes duuidas lhe vem.

Justo he que immagine eu e que estee muyto turbada querer Quem o mundo he Seu, sem merecimento meu, entrar em minha morada e hũa summa Perfeyçam de resplandor guarnecido, tomar pera Seu vestido sangue do meu coração, indigno de ser nacido.

E Aquelle que eccupa o mar, enche os ceos e as profundezas, os orbes e redondezas, em tão pequeno lugar como poderaa estar a Grandeza das grandezas?

GABriel-Porque tanto isto não peses, nem duuides de querer, tua prima Elisabeth he prenhe e de seys meses. 
E tu, senhora, as-de crer que tudo a Deos he possiuel e o que he mais impossiuel lhe he o menos de fazer.

VIRGEM-Anjo, perdoay-me vos, que com a Fee quero falar; pedirey sinal dos ceos.

FeE-Senhora, o poder de Deos nam se ha-de ixaminar.

Nem deueis de duuidar, pois soys d'Elle tão querida.

Anjo-E dabenicio escolhida, e manda-vos conuidar, pera madre vos conuida.

VIRGEM-Ecce ancila Domini, faça-se Sua vontade no que Sua diuindade mandar que seja de mi e de minha liberdade ${ }^{4}$.

El primer problema que debe ocupar nuestra atención es el del origen de esta idea extrabíblica de la Anunciación. en que se inspiran Torres Naharro y Gil Vicente. Estudiando el auto del poetaorfebre, Joaquim de Carvalho afirma ${ }^{5}$ que la personificación de las virtudes es invención de Gil Vicente, pero añade que cabe admitir la posibilidad de que se haya inspirado en un pasaje del Catalogus Sanctorum, vitas, passiones et miracula ... de "Petrus de Natalibus", publicado en Lyon en 1534. Allí se lee lo siguiente:

... Desponsata autem virgine, ipse quidem in ciuitatem suam Bethleem rediit: domum suam dispositurus. Virgo autem Maria cum quinque virginibus coeuis suis: quas ob ostensionem miraculi a sacerdote acceperat in socias ad domum parentum in Nazareth reuersa est. Nomina virginibus erant hec: Rachel: Sephora: Susanna: Abigera et Agabel: quarum omnium Maria etate minor erat: quibus data sunt ad operandum pro vestibus templi sericum: hiacynthus: coccinus: byssus: linum et purpura: ut quelibet ex uno horum vestem contexeret. Cum ergo iactatis sortibus que ipsarum quid horum operari deberet: et Maria sorte purpuram ad velum templi domini accepisset: alie virgines ei dixerunt admirantes: quod cum ipsa minor omnibus esset:

4 Copilaçam, fol. XXII $\mathrm{r}^{\circ}-\mathrm{v}^{\circ}$.

" "Os sermões de Gil Vicente e a arte de pregar", en Joaquim de Carvalho, Estudos sobre a cultura porluguesa do século xvi, vol. II, Coimbra, 1948, págs. 341-342. Sobre este trabajo véase nuestro estudio Les sermons de Gil Vicente. En marge d'un opuscule du professeur Joaquim de Carvalho, Lisbonne, 1949. 
purpuram que ad reginas operari pertinet accepisset. Ideo congruum erat quod ipsa regina virginum vocaretur. Et exinde quasi diuino instinctu ceperunt illam reginam virginum appellare. In diebus autem illis eidem Gabriel angelus oranti apparuit: et de ea nasciturum Dei filium nunciauit: ut dictum est supra in eius annunciatione octaua Kalen. aprilis. Hec Hieronymus.

Conocida es la realidad que se oculta tras este hec Hieronymus. En la época medieval había aparecido el Evangelio de la natividad de Maria, revisión ortodoxa de dos escritos apócrifos, el Protoevangelio de Santiago y el Evangelio del pseudo-Mateo. La revisión, según un procedimiento muy favorecido, se escudó bajo el nombre de San Jerónimo'. Todavía Erasmo, al editar las obras de este santo, aceptaría como auténtico el Evangelio de la natividad de Maria, que había tenido ya una larga carrera. Así, pues, Gil Vicente no tenía ninguna necesidad de recurrir al Catalogus de 1534. Y, por otra parte, se puede afirmar sin riesgo de error que el texto citado no tiene que ver gran cosa con el Auto da Mofina Mendes.

El primer origen del tema tratado por Torres Naharro y Gil Vicente se encuentra en una obra atribuída al gran renovador de la piedad cristiana en la Edad Media, las Meditationes vitae Christi del pseudo-Buenaventura. En el capítulo v (Quo modo Beata Virgo visitavit Elisabeth...), al relatar el viaje de la Virgen a casa de su prima Isabel, el pseudo-Buenaventura precisaba: ... Vadunt autem cum ea paupertas, humilitas et verecundia, omniumque virtutum honestas.

Según su hábito, Ludolfo de Sajonia el "Cartujano" copió este pasaje en su célebre Vita Christi. Y el tema inspiró a los artistas y escritores peninsulares. Hasta los que no sabían latín podían conocer fácilmente ese episodio, pues las Meditationes se publicaron en castellano en 1512 , y la Vita Christi se tradujo al castellano y al portugués.

La fortuna del tema en la pintura portuguesa ha sido estudiada por J. da Costa Lima ${ }^{\top}$. En la Visitación del Maestro de San Francisco, tres de las virtudes del pseudo-Buenaventura están representadas por personajes cuyas inscripciones (Castitas, Paupertas, $\mathrm{Hu}$ militas) nos revelan su identidad. En las Visitaciones del Maestro

"Véase Danill-Roys, Les évangiles de la Vierge, Paris, 1948, págs. 58-59. En las págs. $154^{-155}$ de la misma obra se encontrará una traducción del pasaje correspondiente al que cita Joaquim de Carvalho.

" "Fontes místicas dos pintores quinhentistas", en $B M A A, \mathrm{I}, 1948$, núm. 3, págs. $134^{-1} 4^{6}$ (reimpresión de un ensayo publicado antes en Bro, XLIII, 1946, núm. 1). 
de Viseu y del Maestro de Abrantes, la Virgen va acompañada de dos personajes femeninos.

Tampoco los poetas fueron insensibles a la sugestión del pseudoBuenaventura. En sus Coplas a reverencia de San Juan Baptista, y del misterio de la santa Visitación que la Reina del Cielo hizo a Santa Isabel ${ }^{8}$, fray Ambrosio Montesino se dirige a la Virgen:

$$
\begin{aligned}
& \text { Fe, caridad y hermosura } \\
& \text { e humildad compañas son } \\
& \text { de ti ...? }
\end{aligned}
$$

Es claro, pues, que la personificación de las virtudes no es invento de Torres Naharro ni de Gil Vicente. Pero también es evidente que los dos dramaturgos peninsulares modificaron el tema transmitido a través del pseudo-Buenaventura: en sus obras las virtudes aparecen, no en la Visitación, sino en la Anunciación.

No deja de ser instructivo un examen comparado de las diversas virtudes que aparecen sucesivamente en los textos del pseudo-Bue-

\begin{tabular}{|c|c|c|c|c|}
\hline $\begin{array}{l}\text { Ps.-Buenaven- } \\
\text { tura }\end{array}$ & $\begin{array}{c}M^{\circ} \text { de s. Fram- } \\
\text { cisco }\end{array}$ & Montesino & Torres Naharro & Gil V'icente \\
\hline aup & Paupertas & Caridad & & Pol \\
\hline Humilitas & Humilitas & Humilda & Humildad & Humild \\
\hline erecundia & & Hermosura & Prudencia & Prudencia \\
\hline Honestas & Castitas & $\mathrm{Fe}$ & Virginidad & Fee \\
\hline
\end{tabular}
naventura, de fray Ambrosio Montesino, de Torres Naharro y de Gil Vicente, y en la Visitación del Maestro de San Francisco:

La Humildad es la única virtud común a todos los testigos del tema. La Pobreza de Gil Vicente prueba que no desconocía el texto de las Meditationes vitae Christi o de la Vita Christi (por lo demás, tenemos otras razones para creer que conocía esta última obra ${ }^{10}$ ). En cuanto a la $F e$, el acuerdo entre fray Ambrosio Montesino y Gil Vicente es menos probatorio, aunque no sea arriesgado suponer que el poeta portugués había leído el Cancionero del franciscano uspañol.

Quizá no sea imposible explicar la existencia de virtudes que no se encuentran en el texto del pseudo-Buenaventura: $F e$ (Gil Vicente y Ambrosio Montesino), Virginidad o Castidad (Torres Naharro y el Maestro de San Francisco), Prudencia (Torres Naharro y Gil Vi-

s La primera edición del Cancionero de diversas obras de fray Ambrosio Montesino es de Toledo, 1508.

9 Menéndez y Pelayo, Antología de poetas liricos castellanos (ed. de Obras completas), vol. V, Madrid, 1944, pág. 342.

${ }^{10}$ En nuestro artículo sobre "La source de la Obra da geraçam humana et de l'Auto da alma", en BHTP, I, 1950, págs. 1-32, hemos dado ejemplos concretos de la utilización de la Vita Christi española por el dramaturgo portugués. 
cente), Caridad y Hermosura (Ambrosio Montesino). Vienen, con mucha verosimilitud, de las célebres páginas que San Bernardo consagró a la Virgen, sobre todo en sus sermones. Véase, por ejemplo, en los Loores de la Virgen Madre (homilía IV), el siguiente pasaje, relativo a la Anunciación:

iQue tu humildad se llene de audacia, y tu pudor de confianza! Es cierto que tu sencillez virginal no debe hacerte olvidar la prudencia, pero ésta joh Virgen prudente! es la única ocasión en que no tienes que temer la presunción: si el pudor te ordena guardar silencio, el celo te obliga todavía más a hablar. Abre, Virgen bienaventurada, tu corazón a la fe...

En el Sermón para la natividad de la Santisima Virgen, San Bernardo explica alegóricamente el viaje de la Virgen a través de las montañas para saludar a Santa Isabel:

Verdaderamente ha subido las montañas, ella cuya justicia se eleva como las montañas de Dios [Salmo XXXV, 7]. La Virgen se elevó hasta ellas por tres grados, con ayuda de una triple cuerda difícil de romper [Eclesiastés, IV, 12]: el fervor de la caridad en la búsqueda de la gracia, el esplendor de la virginidad en su carne, la grandeza de la humildad al servicio de su prima ${ }^{11}$.

En cuanto a la Hermosura, podía deducirse de las constantes aplicaciones a la Virgen de pasajes del Cantar de los Cantares.

Con la Prudencia, que aparece a la vez en Torres Naharro y en Gil Vicente, llegamos al terreno de comparación más sólido. Es evidente que, por lo que respecta al tema de la Anunciación, el Diálogo del Nascimiento y el Auto da Mofina Mendes están en relación muy estrecha. Lo que nos permite afirmar categóricamente esta relación no es tanto el hecho de que el tema se traslade de la Visitación a la Anunciación. En las dos obras, el movimiento escénico es el mismo: anuncios sucesivos del Ángel, incertidumbre de la Virgen que se dirige una y otra vez a las Virtudes consejeras y logra por último que le garanticen la autenticidad del mensaje angélico. Es evidente que uno de los dos poetas imitó libremente al otro.

Si nos atenemos a la rúbrica que precede al Auto da Mofina Mendes en la Copilaçam de ${ }_{15}^{62}$ ( $A$ obra seguinte foy representada ao excellente Principe e muyto poderoso Rey dom João terceyro: endereçada aas matinas do Natal na era do Senhor .M.D.xxxiiij), el sentido de la imitación se deduce fácilmente: fué Gil Vicente quien

${ }^{11}$ En estas citas sigo la obra del P. Pie Regamey, O. P., Les plus beaux textes sur la Vierge Marie, Éditions du Vieux-Colombier, Paris, 1946, págs. 127 y 142 (son extractos de las traducciones francesas del P. Aubron, S. J.). 
se inspiró en Torres Naharro, puesto que el Diálogo del Nascimiento se había publicado en 1517 .

Pero, por razones que expondremos en un estudio especial, creemos que la representación de 1534 era reprise de una obra compuesta hacia 1515. A pesar de esta rectificación cronológica, que creemos necesaria, el problema, en opinión nuestra, debe recibir la misma solución. El Diálogo del Nascimiento es, con toda probabilidad, una de las primeras obras de Torres Naharro. Menéndez Pelayo y Wickersham Crawford creían encontrar en la obra una alusión a la batalla de Ravena (11 de abril de 1512); pero el profesor Gillet ha demostrado que la alusión es más bien a las batallas de Cerignola y del Garigliano ( 28 de abril y 28 de diciembre de 1503$)^{12}$.

Así, pues, el texto era ciertamente accesible cuando se produjo el contacto entre el teatro de los dos escritores, o sea en la época de la embajada de Tristan da Cunha a Roma en 1514. Se sabe que, con esta ocasión, Torres Naharro hizo representar una obra de circunstancias, la Comedia Trofea, encargada por el embajador portugués. En otro estudio ${ }^{13}$ he detallado las sugestiones que el poeta-orfebre portugués sacó de este primer contacto: a ellas se puede añadir ahora el tema estudiado en el presente artículo. Si Gil Vicente partió de la imitación de la égloga salmantina de Juan del Encina y Lucas Fernández, no fué insensible, a partir de 1514 , a la influencia del teatro, mucho más desarrollado, de Bartolomé de Torres Naharro.

Institut Français au Portugal.

I. S. RÉVAH

12 "Propalladia”..., op. cit., vol. III (Notes), Bryn Mawr, 1951, págs. 169-17o.

13 "La comedia dans l'œuvre de Gil Vicente", en BHTP, II, 1951, págs. 1-39. 